\title{
Studien(fach)wahlen im Kontext von Klasse und Geschlecht: eine Verknüpfung
}

\subsection{Geschlecht mit Bourdieu gedacht}

Studien(fach)wahlen auf der Grundlage des Habituskonzepts zu analysieren, bedeutet - wie gezeigt wurde -, sie im Rahmen einer beidseitigen Passungskonstellation von Individuum und Hochschule bzw. Fachkultur zu betrachten. Damit geht insbesondere auch die Antizipation eines entsprechendes Berufsfeldes und der damit verbundenen sozialen Positionierung einher. Um diese das Geschlecht einschließende Perspektive theoretisch wie empirisch nutzbar zu machen, soll im Folgenden das Bourdieusche Verständnis von Geschlecht erarbeitet und kritisch reflektiert werden.

Die Analyse von Geschlechterverhältnissen ist bereits in Bourdieus Untersuchungen der kabylischen Gesellschaft enthalten (Bourdieu 1976), in den Mittelpunkt stellt er diese allerdings erst mit seinem Aufsatz (Bourdieu 1997a) und der Monographie (Bourdieu 2005) zur ,,männlichen Herrschaft“. Gleichzeitig spielt Geschlecht in seinen Analysen immer wieder eine Rolle: Bourdieu beleuchtet die ungleichen Bildungschancen zwischen Mädchen und Jungen (Bourdieu/Passeron 1971: 23 ff.) ebenso wie die Bedeutung von heterosexuellen Heiratsstrategien zur Sicherung des sozialen Status (Bourdieu 1981: 198 f.), er blickt auf vergeschlechtlichte Fragen des Geschmacks (Bourdieu 1982: 599) und natürlich auf die geschlechtliche Arbeitsteilung (ebd.: 185; Bourdieu 2005: 163). Dabei geht es in seinen Ausführungen weniger um das Prinzip der Zweigeschlechtlichkeit an sich und die damit verbundenen binären und doch vielfältigen Männlichkeits- und Weiblichkeitspraktiken, sondern es geht ihm stets um die hinter dem Geschlechterverhältnis verborgenen Herrschaftsmechanismen (Krais 2006: 17). 
Analog dazu versteht er die „männliche Herrschaft“ (Bourdieu 2005: 70) als spezifische Form der symbolischen Herrschaft, die Männern systematisch zum Vorteil bekommt: „Manifest wird der den Männern universell zuerkannte Vorrang um einen in der Objektivität der sozialen Strukturen und der produktiven und reproduktiven Tätigkeiten, die auf einer geschlechtlichen Arbeitsteilung der biologischen und sozialen Produktion gründen, welche dem Mann den besseren Part zuweist, und zum anderen in den allen Habitus immanenten Schemata“ (ebd.: 63). Die Rolle der Frauen bei dieser ,sanften, unsichtbare[n], unmerkliche[n] Diskriminierung“ (Bourdieu 1997b: 228) sieht Bourdieu darin, dass ,alle sozialen Zwänge (...) sie [die Frauen] dazu bringen, das Verhältnis männlich-weiblich zu inkorporieren und zu verinnerlichen“ (Bourdieu 2006: 199). So nimmt „... der Beherrschte den Herrschenden mittels Kategorien wahr, die von der Herrschaftsbeziehung hervorgebracht wurden und von daher im Interesse des Herrschenden liegen“ (Bourdieu 1998: 197), während Männern die Herrschaft „,aufgezwungen“ wird (Bourdieu 2005: 195). In der Folge kommt es zu einer umfassenden „Unterbewertung des symbolischen Kapitals, die die Weiblichkeit mit sich bringt" und dazu, dass Frauen ,von den Männern durch einen negativen symbolischen Koeffizienten getrennt sind“ (ebd.: 161 f.). Und auch wenn sich Frauen in den letzten Jahren in vielen Feldern etablieren konnten und zunehmend führende Positionen besetzen, bleibt die „Struktur der Abstände“ zwischen den sozialen Positionen von Frauen und Männern weiter bestehen, was Bourdieu mit der Analogie des „Handikaprennens" illustriert (ebd.: 158 f.). Frauen besetzen nach wie vor in der Tendenz eher unsichere und niedrige Berufspositionen, sie dominieren in schlecht vergüteten Feldern wie den meisten sozialen Berufen und wenn sie Herrschaftspositionen einnehmen, dann sind diese überwiegend in den ,untergeordneten Regionen des Feldes der Macht" angesiedelt - etwa im Journalismus, den Medien oder dem Unterrichtswesen (Bourdieu 1997b: 226 f.) ${ }^{1}$

Aufrechterhalten werden diese Prinzipien der gesellschaftlichen Organisation im Sinne der „männlichen Herrschaft“ durch die bereits erwähnte Verinnerlichung der sozialen Zwänge auf Seiten der Individuen - also durch den Habitus, den Bourdieu als ,,vergeschlechtlicht und vergeschlechtlichend“ (Bourdieu 1997a: 167) beschreibt: Er ist sozial vorstrukturiert, da sich die sozialen Herrschaftsund Ausbeutungszwänge in ihn eingelagert haben, und durch ihn und die mit ihm inkorporierten Muster der Wahrnehmung, Bewertung und Handlung wird Geschlecht hergestellt. Diese vergeschlechtlichten und vergeschlechtlichenden

\footnotetext{
${ }^{1}$ Die Parallelen zu Wetterers professionssoziologischen Erkenntnissen (2002) und zu den strukturtheoretischen Ansätzen der Geschlechterforschung (etwa Becker-Schmidt 2008) sind unverkennbar.
} 
habituellen Dispositionen begründen etwa die größere Nähe von Frauen zu Dienstleistungsberufen oder ihre soziale Zuständigkeit für die Reproduktion von sozialem Kapital - beispielsweise, in dem sie die Familienbeziehungen aufrechterhalten. Gleichzeitig kommt hier die Bedeutung der sozialen Klasse ins Spiel, die sich gleichfalls in den Habitus einlagert: So führe nach Bourdieu etwa eine Arbeiterin Dienstleistungstätigkeiten ,weniger verschleiert, veredelt“ (Bourdieu 1997b: 222 f.) aus als eine Aristokratin. Während eine amerikanische Frau der Mittelklasse mit den Verwandten beider Familien telefoniere, versende eine Frau der Oberklasse Einladungen für Empfänge - im Resultat sieht man geschlechtsspezifische Dispositionen in klassenspezifischem Gewand. Die weiblichen Dispositionen sind demnach unterlegene Dispositionen im Vergleich zu den Männern der gleichen Klasse. Sind aber überlegene im Vergleich zu anderen weiblichen Dispositionen (ebd.) und es kann nicht von den weiblichen Dispositionen gesprochen werden. Vielmehr bieten sich ,,[d]ie Dinge (...) als machbare oder nicht machbare, selbstverständliche oder undenkbare, normale oder ungewöhnliche für diese oder jene Kategorie d. h. insbesondere für einen Mann oder eine Frau (in dieser oder jener Lage) dar" (Bourdieu 2005: 101). Geschlecht und Klasse spielen untrennbar zusammen in der Konstitution sozialer Ungleichheit oder mit Bourdieus Worten: „Die geschlechtsspezifischen Merkmale sind ebenso wenig von den klassenspezifischen zu isolieren wie das Gelbe der Zitrone von ihrem sauren Geschmack“ (Bourdieu 1982: 185).

Das spiegelt sich auch im Habitus wider, in dem beide Dimensionen miteinander verwoben sind. In einem Vergleich bezeichnet Bourdieu das Geschlecht als eine ,ganz fundamentale Dimension des Habitus, die, wie in der Musik die Kreuze oder Schlüssel, alle mit den fundamentalen sozialen Faktoren zusammenhängenden sozialen Eigenschaften modifiziert" (Bourdieu 1997b: 221 ff.). Dabei neigt Bourdieu ,zu der Annahme, daß man lernt, eine Frau zu sein, aber man lernt immer gleich, Tochter oder Frau eines Arbeiters, Tochter oder Frau eines leitenden Angestellten zu sein. (...) Die Geschlechtssozialisation ist von der Sozialisation für eine soziale Position nicht zu trennen“ (ebd.). Im gleichen Interview konkretisiert er kurz darauf seine Vorstellung vom Verhältnis von Klasse und Geschlecht - und dies mag ein Grund sein, warum ihm die Frauen- und Geschlechterforschung regelmäßig mit Kritik oder zumindest Skepsis begegnet: „Mir scheint, die Klassensozialisation ist grundlegend, selbst wenn sie zutiefst von der Geschlechtssozialisation beeinflußt wird“" (ebd.: 224 f.). Doch Bourdieu relativiert schließlich seine Einschätzung und schließt:

„Aber vielleicht müssen wir dieses Problem schlicht und einfach fallenlassen, weil wir nicht die Mittel haben, es zu entscheiden: Was wir beobachten, das sind immer 
gesellschaftlich und geschlechtlich konstruierte Habitus. (...) Man kann wissenschaftlich nicht auseinanderhalten, was der Klasse und was dem gender zukommt. Das ist eine Frage, die ideologische Interessen ins Spiel bringt. Was in der Sozialisation, d. h. in einer vergeschlechtlichten, geschlechtlich bestimmten sozialen Position erworben wird, das ist eine vergeschlechtlichte, geschlechtlich spezifizierte soziale Disposition“ (Bourdieu 1997b: 224 f.).

Denkt man Geschlecht mit Bourdieu, ist es also eine fundamentale Ungleichheitsdimension, die sich zeitgleich mit der sozialen Klasse in den Habitus einschreibt und ihn prägt. Im Ergebnis gibt es einen Habitus, der beide Dimensionen umfasst und die sich analytisch nicht voneinander trennen lassen. ${ }^{2}$

Was die sozialwissenschaftliche Geschlechterforschung anbelangt, „stellte Bourdieus Werk eine Herausforderung dar" (Schlüter/Faulstich-Wieland 2009: 211). Nichtsdestotrotz hat seine Arbeit prägenden Eingang in einige ihrer Ansätze gefunden: So betont Beate Krais die ,ganze Reihe von Affinitäten zwischen seinem analytischen Zugang zur sozialen Welt und feministischen Ansätzen einer ,geschlechtssensiblen“ Gesellschaftstheorie“ (Krais 2001: 318). Sie konkretisiert das Konzept des Habitus als vergeschlechtlichten und vergeschlechtlichenden und stellt heraus, dass die Verinnerlichung der geschlechtlichen Arbeitsteilung als Kernstück der Vergeschlechtlichung des Habitus gefasst werden kann (Krais/Gebauer 2002: 49; Krais 2006: 20). Auf diesem „banalen Element der Arbeitsteilung (...) [baue] eine symbolische Ordnung auf, die hochkomplex, differenziert und enorm variationsreich ist" (Krais 2006: 20) und die bei Männern wie Frauen ,in Fleisch und Blut übergegangen“ und „zur eigenen ,Natur““ (Krais 1993: 217) geworden ist. Zugleich löst laut Krais das Habituskonzept die geschlechtertheoretische Entgegensetzung von ,sex ' und ,gender' als biologisches und soziales Geschlecht auf (Kessler/McKenna 1985 [1978]): So ist der Habitus Speicher sozialer Erfahrung und damit das „Körper gewordene Soziale“eine Dichotomisierung von biologischer und körperlicher Dimension sei müßig (Dölling/Krais 2007: 18 ff.). Und schließlich weist Krais auf die möglicherweise widersprüchlichen Anteile im Habitus hin, denn gerade mit Blick auf Klasse und Geschlecht ,wird deutlich, daß im Zusammenwirken dieser beiden Dimensionen eine Fülle von Reibungspunkten, von heterogenen Erfahrungen für das Subjekt angelegt sind“ (Krais 1993: 220).

\footnotetext{
${ }^{2}$ So wird auch im empirischen Teil dieser Arbeit Geschlecht als Dimension des Habitus gefasst. Dies impliziert durchaus ein sozialkonstruktivistisches Geschlechterverständnis (vgl. West/Zimmerman 1987), das durch den Bourdieuschen Blick allerdings strukturell rückgebunden und intersektional verwoben wird.
} 
Hier schließen auch die an Bourdieu anknüpfenden Arbeiten von Steffani Engler an, die etwa bei der Analyse von studentischen Lebensstilen feststellt, dass Geschlecht einmal in den Vorder- und einmal in den Hintergrund tritt - nämlich je nachdem, ob es sich um ein ,körpernahes“ Merkmal handelt oder nicht (Engler 1997; Engler 1993a). Sie modifiziert daher das Bourdieusche Zitat „Wie einer spricht, tanzt, lacht, liest, was er liest, was er mag, welche Bekannte und Freunde er hat usw. - all das ist eng miteinander verknüpft“ (Bourdieu 1992a: 32) folgendermaßen: ,Wie sie wohnt, sich einrichtet und schläft, ist eng verknüpft mit dem jeweiligen Studienfach; was sie gerne ißt und wo sie ihre Kleidung erwirbt, ist eng verknüpft mit dem Geschlecht“ (Engler 1997: 324). Wie Krais stellt auch Engler heraus, dass sich durch die Denkwerkzeuge Bourdieus Dominanz- und Herrschaftsverhältnisse analysieren und sich dabei Geschlecht in seiner strukturierten und strukturierenden Funktion fassen lässt (Engler 2013: 250 ff.). Doch nicht nur die studentischen Lebensstile sind aus Bourdieuscher Perspektive vergeschlechtlicht: Für das Feld der Wissenschaft allgemein stellt Sandra Beaufaÿs (2003) fest, dass in ihm Mechanismen der Abdrängung wirken, innerhalb derer die Konstruktion des ,leistungsfähigen, individuellen Genies“ eine männliche ist, die zum systematischen Ausschluss von weiblich-vergeschlechtlichten Habitus führe (ebd.: $240 \mathrm{ff}$.).

Kritisch an Bourdieus Arbeiten angemerkt wird von Seiten der Geschlechterforschung wiederum, dass er entscheidende Transformationsprozesse der entwickelten kapitalistischen Gesellschaften ausblende, so etwa eine Aufweichung der Sphären von Öffentlichkeit und Privatheit (Rademacher 2002). Zugleich setzt Claudia Rademacher hier an und verbindet die Bourdieusche Perspektive mit aktuellen Standpunkten der Geschlechterforschung, die strukturalistische Verknüpfungen zulassen, wie jene aus der Professionssoziologie (Wetterer 2002). Dass sich das Habituskonzept entgegen der Vorwürfe des Strukturdeterminismus auch eignet, um sein schöpferisches Erfindungsreichtum $\mathrm{zu}$ analysieren, verdeutlicht im Rahmen der Geschlechterforschung wiederum etwa Flavia Guerrini (2011): Sie arbeitet heraus, dass uneindeutige Geschlechtlichkeiten wie Drag Kings den Blick auf Bewegungen im Habitus ermöglichen.

Dass Bourdieu und die daran anschließenden Arbeiten das Geschlecht nur ungenügend behandeln, wird aus geschlechtertheoretischer Sicht ebenso regelmäßig kritisiert (Koppetsch 2001: 109; Meuser 2006: 114). Dem kann jedoch die Ansicht entgegen gesetzt werden, dass Bourdieu die geschlechtliche Dimension des Habitus durchaus als omnipräsent und fundamental bedeutsam für die Konstitution sozialer Ungleichheit konstruiert. So führen Ulle Jäger, Tomke König und Andrea Maihofer (2015: 24) an, dass Bourdieu seinen sonst zentralen Begriff des Feldes in der ,männlichen Herrschaft“ nicht verwendet und führen das darauf 
zurück, dass die geschlechtliche Dimension des Habitus „für Bourdieu nicht auf ein bestimmtes Feld zu reduzieren [ist], sondern (...) alle gesellschaftlichen Felder gleichermaßen [durchzieht] und (...) damit auch in die Analyse aller sozialen Felder einzubeziehen [ist]". Dieser Deutung folgend ist die Tatsache, dass Bourdieu das Geschlecht nicht in all seinen Arbeiten herausgehoben zum Gegenstand seiner Betrachtungen gemacht hat, kein Zeichen dafür, dass es für seine Theorie zur sozialen Ungleichheit wenig bedeutsam wäre - sondern im Gegenteil dafür, dass Geschlecht in all seinen Analysen sozusagen ständig im Hintergrund ,mitläuft' und als fundamentale Ungleichheitsdimension im Zusammenwirken mit der sozialen Klasse essentielle Bedeutung hat.

Von diesen teilweise kritischen und sonst produktiven Bezügen der Geschlechterforschung auf Bourdieu abgesehen, lässt sich festhalten, dass das geschlechtersoziologische Potential seiner Arbeiten - auch aufgrund des aktuell dominanten poststrukturalistischen Fokus der Geschlechterforschung - noch nicht ausgeschöpft ist. Sein Habitusbegriff, der die Entgegensetzung von körperlicher und sozialer Dimension überwindet und verschiedene Ungleichheitsdimensionen zugleich umfasst, bietet sich geradezu für intersektionale Arbeiten an - nicht ohne Grund weist etwa Helma Lutz in einem Vortrag auf das Potential der Bourdieuschen Perspektive hin, „Analysen von Intersektionalität zu revolutionieren“ (ebd.). Zugleich lohnt es sich, an seinem Verständnis von Klassen als habitusspezifisch handelnde Klassen anzusetzen, um die Bedeutung von Geschlecht dafür herauszuarbeiten. Das soll im Folgenden getan werden.

\subsection{Im Anschluss an Bourdieu: Das Konzept sozialer Milieus}

\subsubsection{Schicht, Milieu, Klasse}

Um die sozialen Logiken von Studien(fach)wahlen im Zusammenspiel von sozialer Herkunft und Geschlecht auf der Grundlage des Habituskonzepts herausarbeiten zu können, bedarf es eines genaueren Blicks auf das ihm zu Grunde liegende Verständnis von ,sozialen Klassen“ ebenso wie einer Abgrenzung von ,sozialen Schichten'.

Soziale Schichten umfassen grundsätzlich Gruppen von Menschen, die durch gleiche oder ähnliche Ausprägungen von einem oder mehreren Merkmalen (Einkommen, Bildung, Prestige) der gleichen definierten ,Schicht" angehören 
(Solga/Berger/Powell 2009: 25 ff.). Empirisch ausgedrückt werden die bundesdeutsche Schichtstruktur und ihre zunehmende Ausdifferenzierung durch verschiedene Modelle wie das „Dahrendorf-Haus“, die „Bolte-Zwiebel“ oder das „Geißler-Haus" - Modelle, denen tendenziell vier Grenzen gesetzt sind (Bremer 2007a: 28): Erstens gehen sie von Berufen, also von der Erwerbssphäre aus, wodurch andere Lebensbereiche wie Freizeit und Lebensform aus dem Blick geraten. Zweitens unterstellen sie - zumindest tendenziell - ein „SeinBewusstseins-Schema“, da sie von der ökonomischen bzw. beruflichen Situation auf das Denken und Verhalten der Menschen schließen. Drittens ermöglichen sie nur eine vertikale Unterscheidung, horizontale Differenzen in der gleichen Schicht können durch sie nicht erfasst werden. Und viertens können durch sie geschlechtliche Ungleichheiten nicht bzw. nicht genügend abgebildet werden (Steinrücke 2006: 71): Zum einen wird durch den ausschließlichen Bezug auf die Erwerbssphäre die geschlechtliche Arbeitsteilung - also v. a. die weibliche Zuständigkeit für die Reproduktionsarbeit - nicht als eigenständige Quelle sozialer Ungleichheit gefasst. Und zum anderen werden als statistische Einheit zumeist ,Haushalte' erhoben, in denen Frauen in einen homogenen Familienstatus eingeordnet werden. Eine differenzierte Betrachtung vergeschlechtlichter oder gar vergeschlechtlichender Soziallagen ist auf dieser Basis nicht möglich.

An diesen Grenzen setzen Milieukonzepte an, die seit den 1970ern davon Abstand nehmen, soziale Gruppen sozial- und berufsstatistisch zu denken und stattdessen auch die alltägliche Lebensweise in den Fokus nehmen (Bremer 2007b: 119): ,Objektive' Faktoren können zwar Hinweise auf bspw. Bildungsentscheidungen sein, aber davon kann nicht auf die Lebenspraxis selbst geschlossen werden - das den Schichtmodellen inhärente Sein-Bewusstsein-Schema wird damit aufgehoben. Durch die explorative Milieuforschung wurde so beispielsweise empirisch gezeigt, dass die Kategorie der ,Arbeiter*innen“ fünf unterschiedliche Typen von Lebensweisen umfasst (ebd.).

Dabei existieren in der Milieuforschung verschiedene Konzepte, die zum Teil auf unterschiedlichen Grundannahmen beruhen. „Empirisch gefunden“ (Bremer 2007a: 29) wurden soziale Milieus erstmals durch die SinusLebensweltforschung: Dieses Milieumodell betont besonders die Ebene der Ästhetik und des sich stärker wandelnden Lebensstils, also die , horizontale Ebene" der Sozialstruktur. In den darauffolgenden Entwicklungen von Milieumodellen kann insbesondere entlang der Linien von Lebensstil- und Ungleichheitsforschung unterschieden werden. Die Lebensstilforschung (etwa Schulze 1992) schließt an die Individualisierungsthese nach Ulrich Beck (1983) an: In diesen Milieukonzepten sind entsprechend die vertikale Dimension sozialer Ungleichheit und sozio-ökonomische Unterschiede wenig bedeutsam. Diese Annahme 
des Bedeutungsverlusts von sozio-ökonomischen Unterschieden teilen die Vertreter*innen der Ungleichheitsforschung (etwa Vester 2001; Bremer/Lange-Vester 2014b) nicht $^{3}$ :

„Soziale Milieus stellen die ganze Lebensweise, die ,Kultur des Alltags“, in den Mittelpunkt. Menschen eines Milieus ähneln sich in ihren Vorlieben und Haltungen zu Arbeit und Bildung, zu Familie und Freunden, zur Freizeit und auch zur gesellschaftlichen Partizipation. Individualisierung und Pluralisierung sind nicht, wie von vielen in Anlehnung an Beck (...) vermutet, gleichzusetzen mit der Auflösung von sozialen Großgruppen. Vielmehr lassen sich (mit vielfältigen Unterschieden im Detail) nach wie vor Gruppen mit ähnlichen Grundhaltungen bzw. mit einem ähnlichen ,Habitus“ finden (...)“ (Bremer 2007a: 28 f.).

Die beiden Milieuansätze stehen sich damit je nach Auslegung mehr oder weniger konträr gegenüber und lassen sich zugespitzt unter den Stichworten der „Determination“ und „Freiheit“ (Bremer/Lange-Vester 2014a: 18) klassifizieren. Mittlerweile hat sich zumindest ein Minimalkonsens zwischen ihnen entwickelt, nach dem es nicht um den Gegensatz völliger Freiheit und völliger Unfreiheit geht und auch nicht um das Primat entweder des Individuums oder der Struktur $(\text { ebd. })^{4,5}$.

Die Vertreter*innen der Ungleichheitsforschung sind dabei zugleich ,Vertreter einer praxeologischen Klassenanalyse" (Vester 2013: 130): Was das Verständnis von sozialer Klasse angeht, grenzt sich Vester etwa von Beck ab und verweist auf zwei mögliche Lesarten der Marxschen Klassentheorie (Vester 2010). So folge Beck dem „offiziellen Marxismus“, der von einer „Kausalkette“ ausgehe, ,in der

\footnotetext{
${ }^{3}$ Vester und andere beziehen sich in ihrer theoretischen Fundierung zum sozialen Milieu u. a. auf Émile Durkheim (2016 [1988]) der das soziologische Konzept sozialer Milieus im Kontext der gesellschaftlichen Arbeitsteilung eingeführt hat (vgl. etwa Vester 2015: 144).

${ }^{4}$ Zuweilen werden sie auch fälschlicherweise ,in einen Topf geworfen“ als ,,Vertreter der Theorien Neuer Sozialer Ungleichheit“, die die Vorstrukturiertheit des Habitus ausblenden und lediglich selektiv seine generative Funktion herausstellen würden, welche die ,Wahl * eines Lebensstils ermögliche (vgl. König 2017: 33) - eine Auslegung, die - wenn überhaupt - nur auf die Vertreter*innen der Individualisierungsthese zutrifft, in keinem Fall aber auf die Vertreter*innen der Ungleichheitsforschung nach Vester (2001), die an den Klassenbegriff von Bourdieu anschließen und den Habitus theoretisch wie empirisch als vorstrukturiert und strukturierend begreifen.

${ }^{5}$ Abgesehen von diesen beiden zentralen Strängen der Milieuforschung finden immer wieder verkürzte bzw. unklare Bezüge auf den Milieubegriff statt oder es werden neue, eigene Milieuansätze entwickelt und empirisch bearbeitet - im Fall der Geschlechterforschung etwa bei Koppetsch (2001), was in Abschnitt 4.2.3 genauer betrachtet wird.
} 
das Politische aus dem Ökonomischen folgt, aus einer mechanischen Abfolge von Krise, Verelendung, Empörung, Eroberung der Staatsmacht und Umgestaltung der Gesellschaft von oben“ (ebd.: 40 f.). Diese Lesart bezeichnet Vester als „subjektlosen geschichtlichen Automatismus“ (ebd.: 34 ff.) und sieht Marx missverstanden. Stattdessen folgt Vester einem akteursorientierten ,alternativen Marxismus“, der von einer „Logik der Praxis“ ausgehe (ebd.: 37 f.): So sind Klassen nicht substantiell und dem Klassenkampf vorgängig, sondern umgekehrt sind soziale Klassen das Resultat von Klassenkämpfen - soziale Klassen erzeugen sich also selbst erst durch ihre Praxis. Mit Edward P. Thompson, der an die historischen Analysen von Marx anknüpft (Vester 2013: 134) und zentraler Bezugspunkt für Vesters Klassenverständnis ist, lässt sich dieses auf den Punkt bringen: „Ich betrachte Klasse nicht als eine ,Struktur' oder gar als eine ,Kategorie', sondern als etwas, das sich unter Menschen, in ihren Beziehungen, abspielt (...)“ (Thompson 1987: 7).

Dieses relationale und praxeologische Klassenverständnis setzt auch Bourdieu an, gleichwohl er sich nicht explizit auf die unorthodoxe marxistische Lesart bezieht (Vester 2013: 138) ${ }^{6}$. So sind Klassen als „Ensembles von Akteuren mit ähnlichen Stellungen“ für ihn lediglich „Klassen auf dem Papier“ und „von theoretischer Natur“, also „keine reale[n], effektive[n] Klasse[n]“, sondern vielmehr ,wahrscheinliche Klasse[n]“ (Bourdieu 1985: 12). Stattdessen legt Bourdieu ein praxeologisches Klassenverständnis an, in dem der Habitus zugleich „Erzeugungsprinzip“ wie auch „Klassifikationssystem“ ist (Bourdieu 1982: 278).

Wie für Bourdieu die Praxis der Akteur*innen - ihr Habitus - maßgeblich ist für die Konstitution sozialer Klassen, ist sie es ebenso für die Konstitution sozialer Milieus: So konnten Vester und andere eine „Brücke zwischen Milieuforschung und Habitustheorie schlagen“ (Müller/Zimmermann 2018: 3), indem sie den Habitus als „milieustiftendes Prinzip“ begreifen (Bremer 2004: 47), da er in den Milieus erworben wird und diese gleichzeitig durch den Habitus der Akteur*innen konstituiert werden. Der Habitus entwickelt sich von der Kindheit an in der Auseinandersetzung mit dem sozialen Umfeld und verweist auch Jahre danach noch auf die Bedingungen, die ihn hervorgebracht haben:

\footnotetext{
${ }^{6}$ Stattdessen grenzt sich Bourdieu von Marx ab: Dabei unterstellt er ihm ein substantielles Klassenverständnis (Bourdieu 1985: 9), das „konstruierte und reale Klasse gleich[setze]“ und eine Unterscheidung zwischen „Klasse-an-sich“ und „Klasse-für-sich“ vornehme und diese in übergangsartige Beziehung zueinander setze. An dieser Stelle nimmt Bourdieu also Abstand vom ,offiziellen Marxismus‘ und beruft sich dabei auf einen vermeintlich marxistischen Begriff (,Klasse-an-sich“), der so von Marx allerdings nie benutzt wurde, wie Vester in einer Analyse dessen Gesamtwerks zeigt (Vester 2008).
} 
„Der Habitus führt als eine Art Wegweiser durch das Leben. Dass dies überwiegend unbewusst und wie automatisch geschieht, wird erst auf der Grundlage bestimmter, langfristig eingeübter Sichtweisen möglich, die auf die im Herkunftsmilieu gegebenen Bedingungen verweisen und die im Laufe der Zeit mit zunehmend schlafwandlerischer Sicherheit praktiziert werden“ (Bremer/Lange-Vester 2014a: $13)$.

Die im sozialen Umfeld vorgefundenen Ressourcen sind damit die Handlungsvoraussetzungen für die Aneignung der eigenen Möglichkeiten und Grenzen (Lange-Vester/Teiwes-Kügler 2013a: 149) und der Habitus spiegelt nicht einfach die ökonomischen Bedingungen wieder, sondern ist „das Ergebnis von relativ autonomen Sozialisationsprozessen in den Milieus“ (Vester 2015: 147). Nach Vester und anderen sind soziale Milieus demnach Gruppen mit ähnlichem Habitus (Vester 2001: 24 f.) und im entsprechenden Modell sind die sozialen Milieus nicht nach der sozialen Position aufgrund ihrer Kapitalverteilung positioniert wie etwa Bourdieu soziale Position im sozialen Raum verortet -, sondern nach dem Typ ihres Habitus (der wiederum eng verbunden ist mit dem Vorhandensein von Kapitalien) (Bremer 2004: 54).

Zusammenfassend machen mehrere Aspekte das Milieukonzepts nach Vester und anderen besonders anschlussfähig für die vorliegende Arbeit: Erstens legt das Vestersche Konzept ein akteursorientiertes und praxeologisches Verständnis von ,sozialen Klassen' an und nimmt damit Abstand von einer substantiellen Definition, die soziale Klassen als „wirklichkeitsferne Artefakte“ (Vester 2013: 130) erscheinen lässt. Zweitens berücksichtigt das Vestersche Konzept - etwa im Gegensatz zur Sinus-Milieuforschung - im Besonderen die vertikale Dimension von Ungleichheit, die das Gefüge sozialer Positionen sowie die mit ihm verwobene ,männliche Herrschaft' umfasst. Drittens geht das Vestersche Konzept über ebendiese vertikale Dimension hinaus, indem es - im Gegensatz zu Modellen sozialer Schichtung - auch die horizontale Ebene der Alltagskultur umfasst. Und viertens ist es nach Vester und anderen der Habitus, der die Angehörigen eines Milieus miteinander verbindet, was wiederum auf das Potential des Habituskonzepts für die Analyse vergeschlechtlichter Praktiken verweist (wie es in Abschnitt 4.1 herausgearbeitet wurde). Deshalb wird im Folgenden zuerst die empirisch erhobene Milieulandkarte nach Vester vorgestellt, um das Konzept sozialer Milieus anschließend systematisch mit der Dimension des sozialen Geschlechts zu verknüpfen. 


\subsubsection{Milieulandkarte nach Vester}

Auf der Grundlage der dargestellten Verbindung von Habitus und sozialem Milieu wurde die Landkarte der sozialen Milieus entwickelt (s. Abbildung 4.1), die das Ergebnis von mittlerweile über 900 qualitativen Interviews ist, die typologisch ausgewertet und mit repräsentativen quantitativen Datensätzen trianguliert wurden (Lange-Vester/Teiwes-Kügler 2013a: 154).

Dieser Logik des Milieumodells folgend können fünf „Traditionslinien“ von sozialen Milieus unterschieden werden, die sich als genealogische Linien verstehen lassen und in denen frühere Klassenstrukturen weiterhin erkennbar sind - sie sind also historisch lang gewachsen und deshalb (relativ) konstant (Lange-Vester/Teiwes-Kügler 2013a: 154). Die Traditionslinien von ,akademischer Intelligenz“ und „Macht und Besitz“ unterscheiden sich insbesondere durch die unterschiedliche Ausrichtung ihrer Reproduktionsstrategien: Während diese in der Linie von Macht und Besitz eher am Erwerb von sozialem Kapital orientiert sind und mit hierarchiegebundenen Habitusmustern einhergehen, geht es in der Linie der akademischen Intelligenz stärker um den zunehmenden Erwerb von kulturellem Kapital und um die damit verbundenen wachsenden Autonomieansprüche (ebd.: 150). Zugleich wird in der Traditionslinie von Macht und Besitz ein stärker repräsentativer Lebensstil verfolgt und mehr Wert auf Disziplin, Ordnung, Autorität und die Einordnung in Hierarchien gelegt - in der Traditionslinie der akademischen Intelligenz dagegen stehen stärker idealistische Ziele im Fokus und es wird eine Abgrenzung von Macht- und Konkurrenzstreben vorgenommen (tendenziell im Milieumodell, rechts oben' zu finden). Beide Traditionslinien oberhalb der Distinktionslinie verbindet wiederum ihr Herrschaftsanspruch und ihre exklusive Lebenspraxis, sie pflegen einen ,feinen“ und individuellen Lebensstil und gehen auf Distanz zum ,gewöhnlichen' Geschmack. Im mittleren Bereich des Milieumodells lässt sich zwischen der "ständisch-kleinbürgerlichen“ Traditionslinie und der Traditionslinie von „Facharbeit und praktischer Intelligenz“ unterscheiden: In diesen „respektablen Milieus“ sind Karriere und Aufstiegsstreben keine zentralen Motive, sondern eher schrittweise Strategien, um sich in Bildung und Beruf weiterzuentwickeln. Beide streben nach sozialer Anerkennung und geachteten, stetigen Lebensweisen. Die Ständisch-Kleinbürgerlichen wiederum orientieren sich dabei stärker an der Einbindung in hierarchische Strukturen, an Status und an Prestige. Die Milieus der Facharbeit dagegen setzen auf Autonomie, Leistung und Kompetenz. Dementsprechend finden sich in der ständisch-kleinbürgerlichen Linie vor allem kleine Beschäftigte, Selbstständige und Angehörige traditioneller Berufe mit materiell und kulturell eher geringen Ressourcen. Sie suchen Sicherheit in der Familie, in der Arbeit und der Politik. 


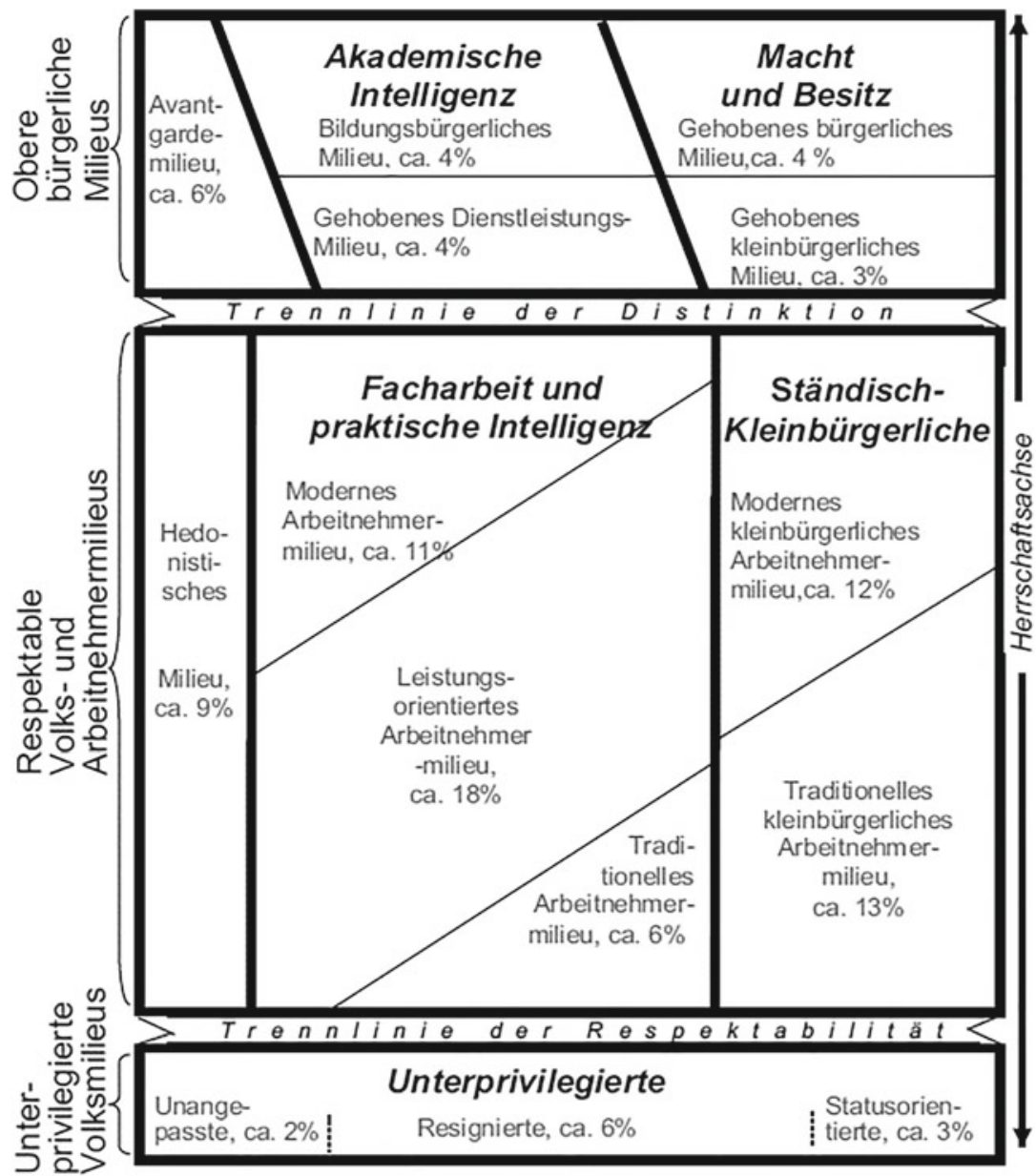

selbstbestimmt

hierarchiegebunden

Differenzierungsachse

Statistische Grundlage der Milieuprofle: Repräsentative Erhebung ( $n=2.699)$ der deutschsprachigen Wohnbevolkerung ab 14 Jahre 1991 (nach: M. Vester u a., Sozale Mǔieus im ge sellschattichen Struktuwandel, Frankfurt a M. 2001); Nauformu ierung der tüheren Milieubezeichnungen aufgrund einer differenzierenden Nouauswertung dieser Erhebung (in: W. Vögele u.a. (Hg.). Soziale Milieus und Kirche. Würthurg 2002); Hochrechnung auf die MilieugröBen von 2003 (nach: Sigma - Sozalwissenschatliches institut für Gegenwartstragen, Die sozialen Mileus in der Verbraucheranalyse, wwwsigma.online.de v. 22.9.2003) - M. Vester (Konzept) / D. Gardemin (Grafik) - agis Universităt Hannover - 2012

Abbildung 4.1 Landkarte sozialer Milieus nach Vester und anderen. (Quelle: TeiwesKügler/Lange-Vester 2018: 118) 
Von den konventionellen, autoritären Habitusmustern grenzen sich die jüngeren Fraktionen der Traditionslinie (etwa die modernen bürgerlichen) zunehmend ab. Generationale Unterschiede finden sich auch in der Traditionslinie der Facharbeit: Die Milieus hier eint ihr Fokus auf kontinuierlicher Weiterbildung, auf Solidarität in der Familie, Nachbarschaft und unter Kolleg*innen und sie folgen einem asketischen Arbeitsethos und zeigen hohe Leistungsbereitschaft. Während sich bei der älteren Generation (dem Traditionellen Arbeitnehmermilieu) allerdings noch ein eher bescheidener Habitus zeigt, hat die jüngere (etwa im Leistungsorientierten Arbeitnehmermilieu) schon enorm an Bildung, Autonomie und Teilhabechancen gewonnen. Die unterprivilegierten Milieus schließlich lassen sich nochmals in drei Gruppen unterteilen: So suchen die „,konservativen Statusorientierten“ Schutz in Hierarchien um ihre Lebenslage zu bewältigen, die ,,arbeitnehmerischen Resignierten“ setzen auf gewerkschaftliche Schutzmacht und die „Unangepassten“ orientieren sich an modernen Lebensstilen, Selbstverwirklichung und teilweise an Bildung (Bremer/Lange-Vester 2014a: 15; Teiwes-Kügler/Lange-Vester 2018: $120 \mathrm{ff}$.).

Auf der Grundlage des Konzepts sozialer Milieus sind Praktiken der Lebensführung in unterschiedlichen Bereichen wie Bildung, Beruf und Familie durch den Habitus miteinander verknüpft und prägen die Konstitution sozialer Ungleichheit. Unter Berücksichtigung der Ausführungen über Geschlecht in Bourdieuscher Perspektive sowie über unterschiedliche Klassenbegriffe und Milieumodelle mit ihrem jeweils spezifischen Verständnis von sozialer Ungleichheit, stellt sich die Frage, wie die Dimensionen von Klasse bzw. Milieu und Geschlecht nun konzeptionell zusammengedacht werden können, um ihr Zusammenwirken in der Entstehung von Studien(fach)wahlen theoretisch und empirisch fundiert zu fassen.

\subsubsection{Geschlecht in der Milieuforschung}

Während die Dimension von Geschlecht im Milieumodell nach Vester konzeptionell inbegriffen ist, steht sie nur einige wenige Male explizit im Interesse dieses Forschungskontextes: So wurden etwa die Strategien der täglichen Lebensführung und der Erwerbsorientierung (Völker 2014; Völker 2004) und die milieuspezifischen Strategien zum Umgang mit der ,Wende' (Lange 1993) von Frauen aus den ,neuen ' Bundesländern herausgearbeitet und es wurde gezielt das Leistungsorientierte Arbeitnehmermilieu beleuchtet, um vergeschlechtlichte Positionen und Einstellungen herauszuarbeiten (Vester/Gardemin 2001).

Ganz grundsätzlich zeigt sich dabei, dass es in allen Milieus etwa gleich viele Frauen wie Männer gibt (Vester 2001: 55), dass es also etwa genauso viele Frauen 
wie Männer des gleichen Mentalitäts- bzw. Habitustypus gibt. Das führt Vester auf den Zusammenhang zwischen dem (statistisch zumeist heterosexuellen) Zusammenleben in Haushalten und ähnlichen Grundhaltungen des Habitus zurück (ebd.: 247). ${ }^{7}$ Gleichzeitig lässt sich in den Milieus eine geschlechtliche Segregation in Form einer vertikalen ,Unterschichtung' von Frauen feststellen, was ihre berufsbezogenen Positionen angeht: So enden etwa die Berufsgruppen von Frauen im Leistungsorientierten Arbeitnehmermilieu ungefähr in der mittleren Höhe des Milieus, „wie an einer imaginären Grenze“ - damit haben „,nur Männer (...) die Chance, den gesamten Berufsraum auszufüllen" (Vester/Gardemin 2001: 480). Zusätzlich werden Frauen in allen Milieus in mehr oder weniger großem Ausmaß bei gleicher beruflicher Qualifikation geringer entlohnt. Die Qualifikation von Frauen wird also bedingt durch die Mechanismen der ,patriarchalen (...) Klassenherrschaft“ ,überproportional abgewertet“ (Vester/Gardemin 2001: 479). ${ }^{8}$ Nach Vester und Gardemin drückt sich so auf der vertikalen Ebene die männliche Herrschaft aus, während auf der horizontalen Ebene zusätzlich die sektorale Arbeitsteilung entlang der Unterteilung in ,produzierende " und ,dienstleistende Tätigkeiten deutlich wird (ebd.: 465 ff.).

Doch nicht nur das - auch die milieuspezifischen Habitus sind vergeschlechtlicht:

„Insgesamt bestätigt die Typologie der Milieus bereits, dass die gesamte Gesellschaft sich nach dem Milieu-Habitus oder, wenn man will, dem Klassen-Habitus gliedert. Auf der Ebene der Alltagsmilieus, d. h. der Ethiken der alltäglichen Lebensführung, haben wir keine tiefgehenden Unterschiede zwischen Männern und Frauen gefunden. Es gibt keine eigenen weiblichen Milieus, und der Anteil der Frauen ist in den Milieus annähernd gleich groß wie der der Männer. Die Unterschiede der Geschlechter beginnen, wie Frerichs und Steinbrücke betonen, erst als Differenzierung innerhalb des Rahmens eines Habitus“ (Vester/Gardemin 2001: 458).

\footnotetext{
${ }^{7}$ Dieser Zusammenhang findet sich so ähnlich auch bei Bourdieu, der die Paarbildung als konstitutives Element der Klassenreproduktion greift (Bourdieu 1982: 734) und die auf der Grundlage von Habitusähnlichkeiten zustande kommt: „Oft schon von Anfang zueinander passend, passen sich die Partner im Zuge gegenseitiger, Akkulturation“ immer noch mehr einander an. Daß ein Habitus sich im anderen wiedererkennt, steht am Ursprung der spontanen Wahlverwandtschaften, an denen soziale Übereinstimmung sich orientiert (...)“ (Bourdieu 1982: 375).

${ }^{8}$ Diese Mechanismen der patriarchalen Klassenherrschaft äußern sich auch darin, dass aus Berufsfeldern, in denen sich Frauen neu etabliert haben, historisch grundsätzlich schlechter gestellte Berufe hervorgegangen sind (Vester/Gardemin 2001: 479) - eine Feststellung und Auslegung, die den professionssoziologischen Erkenntnissen von Wetterer (2002) gleicht.
} 
Und auch wenn es vergeschlechtlichte Habitusanteile gibt, die die Frauen mit Frauen und Männer mit Männern verbinden, können die Autoren einen eigenständigen ,Geschlechtshabitus“ nicht feststellen (Vester/Gardemin 2001: 458). Daher erscheint der Begriff des vergeschlechtlichten und vergeschlechtlichenden Milieuhabitus folgerichtig.

Mit ihrer Untersuchung beziehen sich Vester/Gardemin u. a. auf die Untersuchungen von Petra Frerichs und Margareta Steinrücke, die am Bourdieuschen Verständnis vom Verhältnis von Klasse und Geschlecht ansetzen und die Geschlechtsklassenhypothese und die Klassengeschlechtshypothese aufstellen und empirisch prüfen (Frerichs/Steinrücke 1994; Frerichs/Steinrücke 1993; Frerichs 2000; Steinrücke 2006). Nach der Geschlechtsklassenhypothese ist Geschlecht nicht nur sozialklassenübergreifend konstitutiv für soziale Ungleichheit, sondern es ist ebenso konstitutiv für Klasse - demnach existieren Klassen immer in geschlechtsspezifischen Ausdifferenzierungen, es gibt also „Geschlechtsklassen“ (Frerichs 2000: 40). Nach der Klassengeschlechtshypothese wiederum hat jede Klasse und jede Klassenfraktion eigene Vorstellungen und Praktiken von ,Weiblichkeit“ und ,Männlichkeit', sodass sich Geschlechterbeziehungen klassenspezifisch ausdifferenzieren (ebd.). Mit den aufgestellten Hypothesen wird also nach der ungleichheitsbezogenen Dominanz der Dimensionen von Klasse und Geschlecht gefragt und beide Hypothesen sehen die Autor*innen bestätigt, etwa im Kontext von Erwerbsarbeit (Steinrücke 2006: 78): Hier lassen sich sowohl einkommensmäßige Unterschiede feststellen, die sich in der Dimension von Klasse stärker ausprägen als in der Dimension von Geschlecht, und es offenbart sich zugleich die durchgängige Gemeinsamkeit von Frauen, nämlich hinsichtlich ihrer beruflichen wie einkommensbezogenen Position stets eine Stufe tiefer zu stehen als die Männer ihrer Klassenlage.

Wie genau diese milieuspezifischen und zugleich vergeschlechtlichten Habitus aussehen, machen die Ergebnisse von Frerichs (2000) im Rahmen ihrer Paarforschung deutlich: Anhand von biografischen Interviews mit vier heterosexuellen Paaren aus unterschiedlichen sozialen Milieus (Vester 2001) und ihrer strukturtheoretischen Interpretation im Sinne der objektiven Hermeneutik wurden die Grundmuster der Habitus herausgearbeitet. Hierzu wurden die Frauen und Männer sowohl einzeln wie auch gemeinsam als Paar befragt. Im Ergebnis kontrastiert Frerichs u. a. vier, weibliche' Fälle, die untereinander mehr trennt, als sie verbindet - im Gegenzug verbindet sie mit ihren jeweiligen männlichen Partnern mehr. Die Habitus der Frauen unterscheiden sich z. B. nach der Stärke ihrer Erwerbsarbeitsorientierung, ihrer Einstellung zu Sinn und Ziel von Erwerbsarbeit allgemein, ihrem Lebensstil, ihrem Umgang mit sozialen Kontakten oder ihren Vorstellungen von einem , guten Leben`. Nach Frerichs spricht - wie auch nach Bourdieu 
und Vester - einiges dafür, dass sich die Paare aufgrund von Habitusaffinitäten gefunden haben, denn sie verbinden zentrale gemeinsame Wertpräferenzen, Orientierungen und Geschmacksausprägungen. Diesen qualitativen Zugang, der die Klassengeschlechtshypothese bestätigt, trianguliert Frerichs mit einer quantitativen Auswertung des sozioökonomischen Panels, die ergibt, dass Frauen auch nach diesen Daten den beruflichen und finanziellen Positionen der Männer systematisch vertikal , unterschichtet' sind. Dies deutet Frerichs als Bestätigung der Geschlechtsklassenhypothese (Frerichs 2000: 50 ff.).

Die Habitusanalysen von Steinrücke führen zu analogen Erkenntnissen (Steinrücke 2006): Sie zeigen zudem, wie die befragten Paare durch einen gemeinsamen Habitus verbunden sind. So verbindet das befragte ,Arbeiter*innenpaar" ihre Gemeinschaftsorientierung, ihre Aufmerksamkeit gegenüber dem leiblichen Wohl und ihre Offenheit und Affektivität. Das ,Angestelltenpaar' teilt die Werte der Zweisamkeit und Freiheit, denn beide verfolgen einen großen Freiheitsdrang und stellen die Zweierbeziehung als Medium der Selbstfindung in den Mittelpunkt. Das befragte ,Lehrer*innenpaar" sieht seine Beziehung als Interessensgemeinschaft mit ausgesprochener Sicherheitsorientierung und einer utilitaristischen Auslegung von Beziehungen. Das ,Manager*innenpaar' wiederum teilt eine Erfolgsgemeinschaft: Erwerbsarbeit spielt hier in alle Lebensbereiche hinein, sie teilen einen luxuriösen Geschmack und führen aufgrund der starken Erwerbsorientierung ein geselligkeitsarmes Leben. Zugleich zeigen sich Gemeinsamkeiten qua Geschlecht: So haben die Frauen im Vergleich zu ihren Männern eine unbestimmtere und diskontinuierlichere Erwerbsarbeitsorientierung; bei den Männern ist diese früher festgelegt und geradliniger. Im Klassenvergleich jedoch ist die Erwerbsarbeitsorientierung der Managerfrau viel höher als die des Arbeitermanns - es kann daher mit Bourdieu von ,vergeschlechtlichten Klassenhabitus * gesprochen werden. Auch was den Geschmack und Lebensstil angeht, finden sich geschlechtsspezifische Gemeinsamkeiten, die jedoch kleiner sind als die klassenspezifischen. Auf dieser Grundlage schlussfolgert Steinrücke - und schließt damit an Bourdieu an -, dass die Geschlechtsunterschiede von den Klassenunterschieden überlagert werden (Steinrücke 2006: 81).

Dass nicht nur Geschlecht, sondern auch andere Dimensionen sozialer Ungleichheit mit der Strukturiertheit sozialer Milieus verwoben sind, verdeutlichen die Ergebnisse von Andrea König und Stephan Meise (2013) zur geschlechtlichen Habitusdimension von Migrant*innen unterschiedlicher Milieus. König/Meise betonen das Potential von Bourdieus Ansatz und der daraus entwickelten Milieuperspektive, Ungleichheit intersektional zu begreifen (ebd.: 115 f.). Deshalb arbeiten sie auf der Grundlage des Vesterschen Modells und stellen fest, 
dass das Geschlechterverhältnis in den von ihnen untersuchten drei Milieubereichen - sie kontrastieren prekäre, ,traditionelle" und ,modernisierte ' mittlere Milieus - spezifisch ausgestaltet wird: Eine binäre Arbeitsteilung, die den Mann in den Bereich der Erwerbsarbeit und die Frau in jenen der häuslichen Arbeit verweist, scheint im prekären migrantischen Milieu unhinterfragt - ihr Habitus ist auf die Bewältigung alltäglicher Notwendigkeiten ausgerichtet und legt daher eine pragmatische Aufteilung nahe. Ein großer Einfluss tradierter Geschlechterrollen findet sich auch im ,traditionellen' mittleren Milieu, wobei hier die geschlechtsspezifische Arbeitsteilung zumindest thematisiert und teilweise ausgehandelt wird. Im ,modernisierten " mittleren Milieu wiederum spielen egalitäre Ansprüche eine vergleichsweise große Rolle: Frauen dieser Milieus nehmen eine Diskrepanz zwischen Anspruch und Realität in Sachen Gleichberechtigung wahr und die häusliche Arbeitsteilung liegt teilweise quer zu traditionellen Mustern (ebd.: $119 \mathrm{ff}$.).

Die Dimension von Geschlecht wurde in der Vesterschen Milieuforschung damit sowohl grundlegend berücksichtigt wie auch mehrfach gezielt betrachtet, allerdings eher punktuell und ohne einen umfassenden Rückbezug auf geschlechtertheoretische Erkenntnisse ${ }^{9}$. Auch eine daran anknüpfende Verbindung zu Bildungs- und Berufswegen steht bislang aus. Diese Leerstelle soll durch die vorliegende Arbeit gefüllt werden.

Zur theoretischen Absicherung es lohnt sich, vorab einen Blick auf alternative Milieukonzepte und die dortige Einbindung von Geschlecht zu werfen: Eine Verknüpfung von Geschlecht und Milieu wird anhand des geschlechtertheoretischen Konzepts der hegemonialen Männlichkeit (Connell 2015) vorgenommen, etwa bei Michael Meuser (2006; 2008) und Nina Baur (Baur/Akremi 2011). Baur definiert hegemoniale Männlichkeit als ,hegemoniale Gruppe“ von Männern, die die „Definitionsmacht [hat], welche Männlichkeiten als bevorzugt gelten und mit hohem sozialem Status, Gütern und Frauen ausgestattet werden" (Baur/Akremi 2011: 281). Für Baur ist eine entsprechende Praxis hegemonialer Männlichkeit daher ausschließlich bei statushohen Männern zu finden, die viel Zeit in Erwerbsarbeit investieren, die Organisation von Familienalltag und Konsum dagegen in den weiblichen Zuständigkeitsbereich zuordnen. Nach diesem Verständnis sind Praktiken hegemonialer Männlichkeit eng mit klassenspezifischen Praktiken verwoben - der Vestersche Begriff der ,patriarchalen Klassenherrschaft“" scheint also

\footnotetext{
${ }^{9}$ Susanne Völker (Völker 2004: 153 ff.) arbeitet zwar die Grenzen und Potentiale des Habituskonzepts vor dem Hintergrund dessen feministischer Rezeption heraus - ähnlich wie es hier in Abschnitt 4.1 vorgenommen wurde - und wendet dies anschließend für die Analyse der Habitusmuster von ostdeutschen Frauen im Erwerbssystem an, allerdings ohne einen expliziten Bezug auf des Vestersche Milieukonzept.
} 
auch hier passend (Vester/Gardemin 2001: 479). Für Meuser sind die beiden Perspektiven von Bourdieu und Connell ebenfalls miteinander vereinbar: Dies begründet er erstens damit, dass beide Ansätze eine „doppelte Distinktionslogik“ teilen, die die Dominanzverhältnisse von Männern gegenüber Frauen und Männern gegenüber anderen Männern relevant setzen - Bourdieu spricht hier von der libido dominandi (Bourdieu 1997a: 215). Zweitens stellen sie dabei den Wettbewerb als zentrales Mittel männlicher Vergemeinschaftung heraus (Meuser 2008: 35). Meuser betont, dass sich Ausprägungen von Weiblichkeit und Männlichkeit je nach den „lebensweltlichen Erfahrungshintergründen“ wie dem sozialen Milieu, der Generationszugehörigkeit, der Entwicklungsphase und der familiären Situation unterscheiden und sich der Habitus in Abhängigkeit von diesen „Relevanzstrukturen“ manifestiert (Meuser 2006: 120). Während Bourdieu die Klasse als grundlegende Dimension des Habitus konstatiert (Bourdieu 1997b: 224 f.), legt Meuser also einen leicht abgeänderten Habitusbegriff an und verknüpft ihn zudem konzeptionell mit dem Modell zur hegemonialen Männlichkeit, die er als „Kern des männlichen Habitus“ (Meuser 2006: 123) begreift. Was den Milieubezug angeht, rekurriert Meuser auf einen ,grob umrissene[n] Milieubegriff“, indem er Fallanalysen aus bürgerlichen und Arbeitermilieus kontrastiert und das Milieu im Kontext seiner Bedeutung als „konjunktiven Erfahrungsraum“ fasst (Meuser 2006: 305). Die Bourdieusche Vorstellung von sozialen Klassen als handelnde Klassen findet hier also keinen Anschluss.

Auch an anderer Stelle wird die Anschlussfähigkeit von den Konzepten des Habitus und der männlichen Hegemonie diskutiert: Mechthild Bereswill und Anke Neuber (2013) arbeiten die unterschiedliche Temporalität der beiden Ansätze heraus. Während der Bourdieusche Fokus auf der Reproduktion männlicher Herrschaft und ihrer Beharrungskraft liegt, beschäftigt sich Connells Ansatz mit dem Wandel der Konfiguration hegemonialer Männlichkeit. Zudem legen beide Perspektiven unterschiedliche Schwerpunkte, was die Strukturierung von Ungleichheit angeht: Während im Bourdieuschen Ansatz die männliche Herrschaft zwar die gesamte Gesellschaft durchzieht, aber nur eine spezifische Form der symbolischen Herrschaft im dahinter liegenden Klassengefüge ist, überkreuzt die hegemoniale Männlichkeit nach Connell zwar verschiedene Ungleichheitsdimensionen wie race und class, bleibt aber der zentrale Bezugspunkt für die Strukturierung von sozialer Ungleichheit (Bereswill/Neuber 2013: 92 ff.). Anknüpfungen zwischen Bourdieu und Connell sind also durchaus möglich und bieten sich partiell an, aber diese grundsätzlichen konzeptionellen Unterschiedlichkeiten sprechen dafür, eine entsprechende Verbindung im Folgenden nicht vorzunehmen. 
Auch Cornelia Koppetsch greift in ihrem Milieuverständnis auf Connell zurück, entwickelt allerdings ein alternatives Milieuverständnis und zeigt auf dieser Basis, wie Geschlechterpraktiken und -verhältnisse und insbesondere die geschlechtliche Arbeitsteilung sich milieuspezifisch konstituieren (Koppetsch/Burkart 1999; Koppetsch 2001; Koppetsch/Speck 2015). Ihr Zugang hat mit dem Vesterschen Ansatz gemeinsam, dass sie sich von der Beckschen Individualisierungsthese distanziert und daher nicht von einer Auflösung der Geschlechterrollen ausgeht, sondern von der Verlagerung der dahinter liegenden Reproduktionsbedingungen und der nach wie vor wirksamen Reproduktion der Geschlechterrollen (Koppetsch/Burkart 1999: 6 f.). Bezeichnend für Koppetschs Perspektive auf Milieu und Geschlecht ist, dass sie das Geschlechterverhältnis als konstitutive Struktur für die Definition sozialer Milieus begreift und ,Geschlecht' in gängigen Ansätzen der Milieuforschung nach Schulze und Bourdieu vernachlässigt sieht (ebd.: 11 f.; auch Koppetsch/Speck 2015: 28). Dabei sind nach ihrem Verständnis Praktiken von Erwerbsarbeit und Arbeitsteilung maßgebend für die Konstitution des milieuspezifischen Geschlechterverhältnisses und es genüge nicht, zu zeigen, dass jedes Milieu seine eigenen Vorstellungen von Weiblichkeit und Männlichkeit hervorbringt. Stattdessen müsse das Geschlechterverhältnis ,im Kontext der Gesamtheit der Praktiken [gesehen werden, L.L.] (...), die darauf abzielen (...) [die] Stellung [des Milieus] innerhalb der Struktur des Klassenverhältnisses zu wahren oder zu verbessern“ (Koppetsch 2001: 113). Nach diesem Verständnis ist Geschlecht also konstitutiv für das soziale Milieu.

Diese milieukonstituierende Bedeutung von Geschlecht ist einer der zentralen Erkenntnisgewinne von Koppetschs Studien und soll auch im Folgenden Berücksichtigung finden ${ }^{10}$. Sie ist zugleich anschlussfähig an die Arbeiten Bourdieus, für den sich eine Klasse ebenfalls ,wesentlich“ (Bourdieu 1982: 185) durch Stellung, Wert und die gesellschaftlich ausgebildeten Einstellungen der beiden Geschlechter definiert und im Rahmen dessen sowohl die Arbeitsteilung wie auch die Paarbildung und damit verbundene Praktiken zu den zentralen Reproduktionsstrategien gehören, die darauf abzielen, die Position im Klassenverhältnis zu sichern (ebd.; Bourdieu 1982: 210; Bourdieu 1981: 198 f.) Für Vester wiederum sind Geschlecht und Geschlechterbeziehungen Teil der Klassenbeziehungen (Vester 2001: 159) und auch wenn in diesem Forschungskontext die konstitutive Bedeutung von Geschlecht(erbeziehungen) für die Milieus bislang nicht erforscht wurde, kann daraus nicht geschlossen werden - wie Koppetsch es tut - dass diese Bedeutung negiert würde.

\footnotetext{
${ }^{10}$ Wobei sich diese Erkenntnis auch schon in den Untersuchungen von Frerichs (2000) und Steinrücke (2006) findet, wie dargestellt wurde.
} 
Aus dieser Perspektive auf Geschlecht und Milieu folgt Koppetschs Vorgehen, sich nicht auf ein vorhandenes Milieukonzept zu stützen, sondern anhand von Interviews mit (i. d. R. heterosexuell zusammenlebenden) Paaren deren kulturelle Leitvorstellungen in Bezug auf Bildung und Beruf herauszuarbeiten und so eine eigene grobe Milieudifferenzierung zu entwickelt. Dabei sind die Interdependenzen zwischen den Ebenen von „Beziehungskode“, ,institutionellem Setting“, „Geschlechterkode“ und „Ausdrucksmedien“ entscheidend für die Zuordnung zu einem Milieu (Koppetsch/Burkart 1999: 328). ${ }^{11}$ Im Ergebnis unterscheidet Koppetsch ein traditionales, ein familistisches und ein individualisiertes Milieu und stellt fest, dass die Geschlechternorm von ,partnerschaftlicher Gleichheit“ nur im individualisierten Milieu zu finden ist, wobei diese von den Partner*innen nicht praktiziert wird, sondern nur eine „von beiden Partnern gepflegte Illusion“ ist (ebd.: 317). Diese Erkenntnisse zur milieuspezifischen Arbeitsteilung und zum Widerspruch zwischen Anspruch und Realität egalitärer Geschlechterbeziehungen sind überwiegend deckungsgleich mit den Ergebnissen von König/Meise (2013). Daher sollen im Folgenden diese Erkenntnisse zu Geschlechternormen, insbesondere vor dem Hintergrund der geschlechtlichen Arbeitsteilung, Berücksichtigung finden ${ }^{12}$.

\subsection{Zwischenfazit: Studien(fach)wahlen als Ausdruck vergeschlechtlichter und vergeschlechtlichender Milieuhabitus}

Auf der Grundlage des Habituskonzepts lassen sich die Dimensionen von Klasse und Geschlecht zusammendenken, ohne eine der beiden unterkomplex zu behandeln: So kommen beide im Habitus als opus operatum - als strukturierte

\footnotetext{
${ }^{11}$ Unter Beziehungskode fasst sie die Ausprägungen „,individualisierte Partnerschaft“, „Familismus“ und „Patriarchalismus“; unter institutionelles Setting „Bildungssystem“; „Familie“ und „lokale Netzwerke“; unter Geschlechterkode „Egalität“, „Komplementarität“ und „Hierarchie“ und unter Ausdrucksmedien „Diskurs“, „Atmosphäre“ und „Ritual“ (Koppetsch/Burkart 1999: 328).

${ }^{12}$ Darüber hinaus ist eine Anlehnung an Koppetsch in der vorliegenden Arbeit jedoch schwierig: Indem Koppetsch ihre Definition von Milieus auf den gefundenen Geschlechterpraktiken aufbaut, unterscheidet sich ihr Vorgehen ganz grundsätzlich von einer an Bourdieu angelehnten Milieuforschung, die den klassenspezifischen (und von Geschlecht durchzogenen) Habitus als milieubestimmendes Merkmal heranzieht. Zudem grenzt sich Koppetsch explizit von existierenden Milieutheorien ab (Koppetsch/Burkart 1999: 327) und der Bezug auf eine differenzierte Betrachtung des Klassenbegriffs bleibt aus.
} 
Struktur - und als modus operandi - als strukturierende Struktur - zusammen (Bourdieu 1981: 197). Dieses intersektionale Habitusverständnis - und das Bourdieusche Verständnis von Klasse als handelnde Klasse - ist Basis für das Milieukonzept nach Vester, in dem Geschlecht also konzeptionell inbegriffen ist, zugleich aber bislang nur selten expliziter Gegenstand der Betrachtungen war.

Was soziale Milieus an sich betrifft, so werden diese je nach Ansatz unterschiedlich definiert: über den Habitus (wie nach Vester) oder über Geschlechterpraktiken (wie nach Koppetsch). Der differenzierte Klassenbegriff und das intersektionale Potential des Habituskonzepts wiederum machen das Vestersche Milieumodell an dieser Stelle besonders anschlussfähig. Es wird daher die Grundlage für die empirische Analyse dieser Arbeit bilden.

Geschlechterpraktiken werden dabei - und dies mag der kleinste gemeinsame Nenner zum Milieuverständnis nach Koppetsch sein - so gefasst, dass sie sich milieuspezifisch konstituieren und wiederum zur Konstitution des Milieus selbst beitragen. Indem man der Klassengeschlechtshypothese folgt, nach der es milieuspezifische Männlichkeiten und Weiblichkeiten gibt, und zugleich die Geschlechtsklassenhypothese berücksichtigt, die auf die milieuspezifisch unterschichteten sozialen Positionen von Frauen hinweist (Frerichs 2000; Steinrücke 2006), wird der grundlegenden Verwobenheit der beiden Dimensionen und ihrer gleichermaßen essentiellen Bedeutung für die Produktion sozialer Ungleichheit Rechnung getragen.

Diese Verwobenheit stellt die bevorstehende Analyse vor besondere Herausforderungen. In welchem Verhältnis Klasse bzw. Milieu und Geschlecht zueinanderstehen, wird in verschiedenen Milieubezügen unterschiedlich begriffen - ebenso kommt hier regelmäßig die Frage nach der Dominanz einer der beiden Dimensionen auf. Bourdieu umgeht dieses Problem gewissermaßen, da man nach ihm „...dieses Problem schlicht und einfach fallenlassen [muss], weil wir nicht die Mittel haben, es zu entscheiden“ (Bourdieu 1997b: 224 f.). Es soll auch im Folgenden davon abgesehen werden, nach der grundsätzlichen Dominanz von Klasse oder Geschlecht zu fragen. Für die Habitusanalyse von Studien(fach)wahlen bedeutet das, dass die Fälle stets als Mann bzw. Frau eines spezifischen sozialen Milieus vor dem Hintergrund ihres sozialisatorischen Gewordenseins betrachtet werden. Gleichzeitig gilt es jedoch, die dargestellten Erkenntnisse der Geschlechterforschung zu berücksichtigen und somit den Blick auch auf Vergeschlechtlichungsprozesse des Habitus und der Studien(fach)wahl zu richten - hier stößt in der Theorie postulierte Verwobenheit von Klasse und Geschlecht also durchaus an ihre Grenzen. Dieser Widerspruch im analytischen Vorgehen muss an dieser Stelle ausgehalten werden. 
Zusammenfassend lassen sich Studien(fach)wahlen als Ausdruck von vergeschlechtlichten und vergeschlechtlichenden Milieuhabitus begreifen: Welche (implizite) Vorstellungen von der Lebensführung sowie von Beruf und Familie bei einem Individuum vorhanden sind, ist eng verbunden mit der Sozialisation in einem spezifischen sozialen Umfeld und der Inkorporierung entsprechender milieuspezifischer Muster der Wahrnehmung, Handlung und Bewertung - also milieuspezifischer Habitusmuster. Diese sind im gleichen Zug vergeschlechtlicht: Das Gespür für Bereiche und Tätigkeiten, die einem ,nahe liegen ' und für die Passung zu den daraus resultierenden sozialen Positionen, hängt davon ab, als ,Frau ‘ oder ,Mann' (eines bestimmten sozialen Milieus) habitualisiert zu sein. Deutlich wird damit nicht nur, dass Studien(fach)wahlen in die gesamte Lebensführung eingebettet (Bremer/Lange-Vester 2019), sondern auch, dass sie als Mechanismus der vergeschlechtlichten Arbeitsteilung eng mit den milieuspezifischen Geschlechterpraktiken verwoben sind, die im Sinne ihrer „Brückenfunktion“ (Koppetsch/Burkart 1999: 13) wiederum konstitutiv für das Milieu selbst sind. Welches Studium ,Frau“ ergreift, welche berufliche Tätigkeit sie anschließend ausübt, welche Paarbeziehung sie eingeht und wie sie Familienarbeit organisiert - all das ist eng miteinander verbunden über den vergeschlechtlichten Milieuhabitus.

Auf dieser Grundlage lassen sich Studien(fach)wahlen im Kontext vergeschlechtlichter und milieuspezifischer Passungsprozesse zwischen Habitus und Hochschule bzw. Studienfach und Beruf greifen: Die habitusspezifischen Dispositionen werden in Positionen der sozialen Ordnung transformiert (Bremer/LangeVester 2019) und tragen so zur gleichzeitigen Konstitution von Beruf und Geschlecht inklusive der damit verbundenen Hierarchisierungen bei (Wetterer 2002). Analytisch greifen lassen sich diese Passungsprozesse mit dem Konzept der vergeschlechtlichten und vergeschlechtlichenden Milieuhabitus, nach dem Studien(fach)wahlen nicht als isolierte Entscheidungen, sondern eingebettet in die milieuspezifischen (und milieukonstituierenden) Geschlechterpraktiken eingebettet betrachtet werden. Diese Perspektive soll im Folgenden empirisch angewendet werden. 
Open Access Dieses Kapitel wird unter der Creative Commons Namensnennung 4.0 International Lizenz (http://creativecommons.org/licenses/by/4.0/deed.de) veröffentlicht, welche die Nutzung, Vervielfältigung, Bearbeitung, Verbreitung und Wiedergabe in jeglichem Medium und Format erlaubt, sofern Sie den/die ursprünglichen Autor(en) und die Quelle ordnungsgemäß nennen, einen Link zur Creative Commons Lizenz beifügen und angeben, ob Änderungen vorgenommen wurden.

Die in diesem Kapitel enthaltenen Bilder und sonstiges Drittmaterial unterliegen ebenfalls der genannten Creative Commons Lizenz, sofern sich aus der Abbildungslegende nichts anderes ergibt. Sofern das betreffende Material nicht unter der genannten Creative Commons Lizenz steht und die betreffende Handlung nicht nach gesetzlichen Vorschriften erlaubt ist, ist für die oben aufgeführten Weiterverwendungen des Materials die Einwilligung des jeweiligen Rechteinhabers einzuholen.

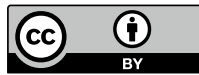

